\title{
Higher Viral Load of Emerging Norovirus GII.P16-GII.2 than Pandemic GII.4 and Epidemic GII.17, Hong Kong, China
}

\author{
Sarah K.C. Cheung, Kirsty Kwok, Lin-Yao Zhang, \\ Kirran N. Mohammad, Grace C.Y. Lui, \\ Nelson Lee, ${ }^{1}$ E. Anthony S. Nelson, \\ Raymond W.M. Lai, Ting F. Leung, \\ Paul K.S. Chan, Martin Chi-Wai Chan
}

We compared viral load of emerging recombinant norovirus GII.P16-GIl.2 with those for pandemic GII.Pe-GII.4 and epidemic GII.P17-GIl.17 genotypes among inpatients in Hong Kong. Viral load of GII.P16-GII.2 was higher than those for other genotypes in different age groups. GII.P16-GII.2 is as replication competent as the pandemic genotype, explaining its high transmissibility and widespread circulation.

$\mathrm{N}$ orovirus, the leading cause of acute gastroenteritis, evolves through mutation and recombination (1). Noroviruses are named by dual nomenclature using the genotype of RNA-dependent RNA-polymerase (RdRp) and major capsid protein (VP1) (2). Recently, 2 recombinant noroviruses carrying RdRp genotype GII.P16 with 2 other VP1 genotypes emerged and spread worldwide: GII.P16GII.4 in the United States and Europe and GII.P16-GII.2 in Europe and Asia in 2016 (3-5). GII.P16 actively recombined with $\geq 8$ capsid genotypes (6-8) and may have pandemic potential and lead to a change in norovirus epidemiology. Phylogenetic and sequence analyses indicated that recent GII.P16-GII.2 had no remarkable change on capsid protein compared with earlier GII.2 strains, suggesting that factors other than immune escape or change in affinity for histo-blood group antigens (i.e., host susceptibility) may play a role in the recent reemergence (6). We compared the viral load of norovirus GII.P16-GII.2 with pandemic GII.Pe-GII.4 and epidemic GII.P17-GII.17 in a cohort of hospitalized patients in Hong Kong over a 5-year period. Our findings may explain, at least in part, the high transmissibility and widespread circulation of GII.P16-GII.2.

\section{The Study}

This study was part of an ongoing molecular surveillance study of norovirus genotype in hospitalized cases in Prince

Author affiliation: The Chinese University of Hong Kong, Hong Kong, China

DOI: https://doi.org/10.3201/eid2501.180395 of Wales Hospital, Hong Kong, during August 2012-June 2017. Norovirus genotype distribution has been detailed in earlier reports $(9,10)$. The fecal norovirus load was determined by a genogroup-specific quantitative real-time reverse transcription PCR (qRT-PCR) assay (11) (Appendix, http://wwwnc.cdc.gov/EID/article/25/1/18-0395-App1. pdf) and was expressed as cycle threshold $\left(\mathrm{C}_{t}\right)$ value that has been demonstrated in a large-scale analysis of CaliciNet data to associate with host and virologic factors (12). A lower $\mathrm{C}_{t}$ value represents a higher norovirus load (Appendix Figure 1). In the data analysis, we stratified cases by 3 patient age groups: $<5$ years, $5-65$ years, and $>65$ years. Continuous variables between 2 and 3 groups were compared by the Mann-Whitney U test and the KruskalWallis test with Dunn's multiple comparison correction, respectively, by Prism 7 for Mac (GraphPad, https://www. graphpad.com/scientific-software/prism). A 2-tailed p value $<0.05$ was considered statistically significant.

During the 5-year period, we collected fecal samples at admission from 1,465 hospitalized patients with laboratoryconfirmed norovirus gastroenteritis. The median age of patients was 3 years (interquartile range [IQR] 1-50 years); male:female ratio was 1:1.1. Norovirus genotype was successfully determined for 1,269 (86.6\%) samples. We excluded $8(0.6 \%)$ patients co-infected with $\geq 1$ norovirus genotype from viral load analysis. The top 3 circulating norovirus genotypes were GII.Pe-GII.4 ( $\mathrm{n}=657 ; 51.8 \%$ ), GII.P17-GII.17 $(\mathrm{n}=191 ; 15.1 \%)$, and GII.P16-GII.2 $(\mathrm{n}=$ $136 ; 10.7 \%)$.

We found that the viral load was higher for emerging GII.P16-GII.2 norovirus than for pandemic GII.PeGII.4 and epidemic GII.P17-GII.17 (Figure, panel A). In young children $<5$ years of age, the median viral load of GII.P16-GII.2 was as high as that of GII.Pe-GII.4 (median $\mathrm{C}_{\mathrm{t}}$ [IQR]: GII.P16-GII.2, 15.2 [12.9-18.8]; GII.Pe-GII.4, 16.7 [14.8-19.0]; $p=0.200$ ). In patients 5-65 years of age, the median viral load of GII.P16-GII.2 was 28 -fold higher than that of GII.Pe-GII.4 and 42-fold higher than that of GII.P17-GII.17. In patients $>65$ years of age, the median viral load of GII.P16-GII.2 was 45-fold higher than that of GII.Pe-GII.4 and 274-fold higher than that of

${ }^{1}$ Current affiliation: Faculty of Medicine and Dentistry, University of Alberta, Edmonton, Alberta, Canada. 
GII.P17-GII.17. The median viral load of GII.Pe-GII.4 declined with age, whereas that of GII.P16-GII.2 remained at the same high level among different age groups. GII.P17GII.17 had the lowest viral load in most comparisons.

We did 2 additional subgroup analyses (sensitivity tests) to validate the robustness of the high viral load observation of GII.P16-GII.2. First, we compared the viral load of cases only during their first season of emergence (i.e., in an immune naive population): for GII.Pe-GII.4, August 2012-June 2013; for GII.P17-GII.17, July 2014-June 2015; and for GII.P16-GII.2, July 2016-June 2017. We observed a trend similar to that of all cases in which viral load of GII.P16-GII.2 was as high as GII.Pe-GII.4 in young children $<5$ years of age and higher than those of GII.Pe-GII.4 and GII.P17-GII.17 in older children, adults, and the elderly (Figure, panel B). Second, we compared co-circulating GII.P16GII.2 and GII.Pe-GII.4 in the last season, July 2016-June 2017, to minimize sample processing variation over time. Again, we observed a trend similar to that of all cases and those during first season of emergence (Appendix Figure 2).

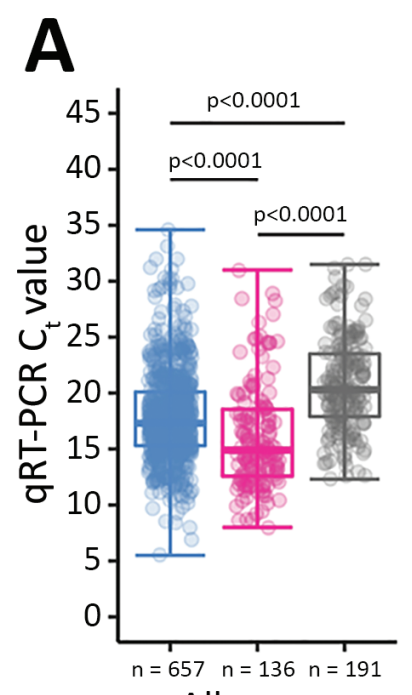

All ages

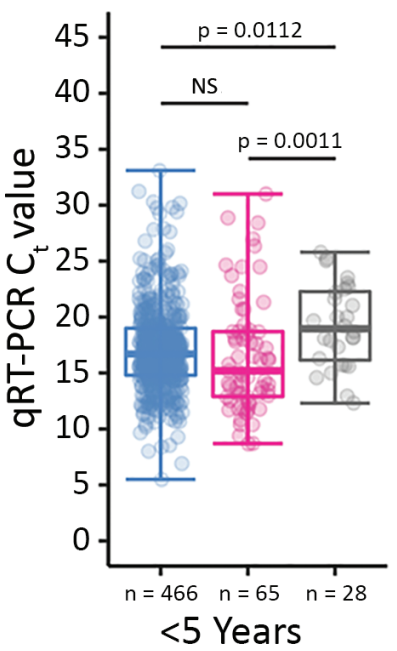

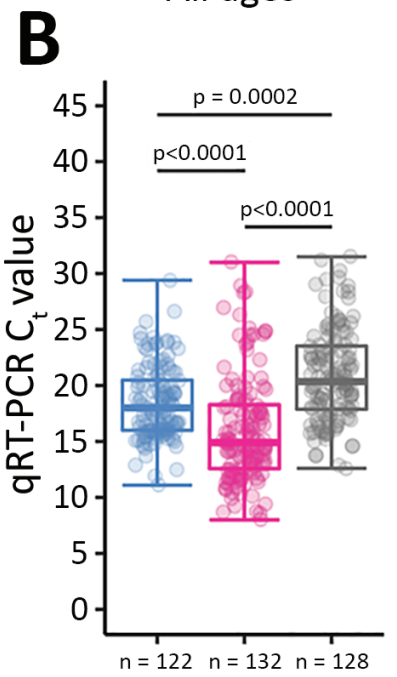

All ages

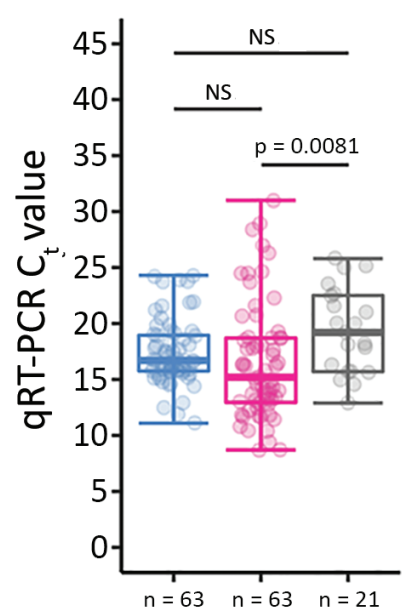

$<5$ Years
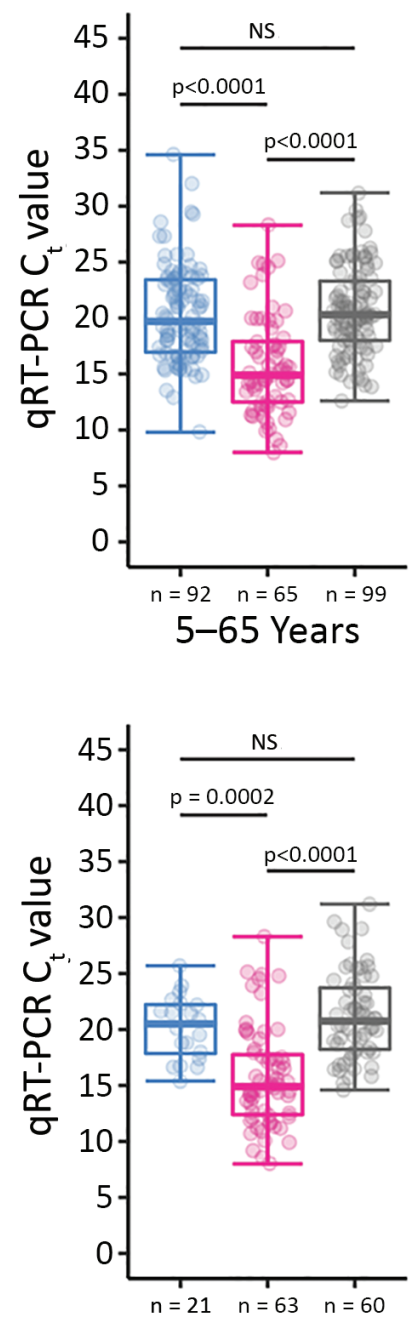

5-65 Years
官GII.Pe-GIl.4

官GII.P16-GII.2

官GII.P17-GII.17
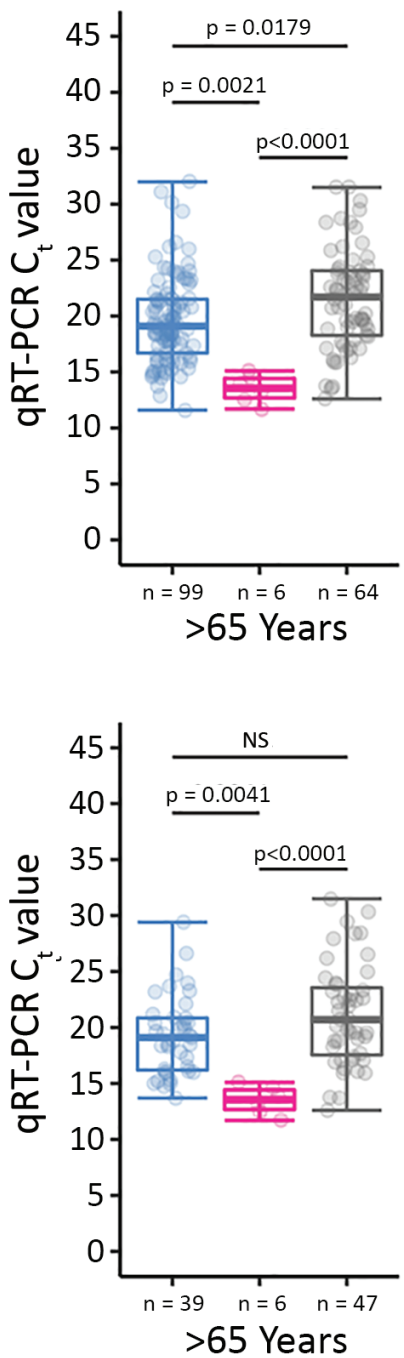

Figure. Higher fecal viral load of recombinant norovirus genotype GII.P16-GII.2 compared with pandemic GII.Pe-GII.4 and epidemic GII.P17-GII.17 among patients in Hong Kong, August 2012-June 2017. A) Results for the whole study period; B) results for the first season of emergence for each genotype: GII.Pe-GII.4, August 2012-June 2013; GII.P16-GII.2, July 2016-June 2017; and GII.P17GII.17, July 2014-June 2015. Data shown are stratified by age group of patients. Each dot represents a patient; box tops and bottoms indicate interquartile range; horizontal lines within boxes indicate medians; error bars indicate maxima and minima. $C_{t}$ values were determined by $q R T-P C R$ and used as proxies for norovirus load. A lower $C_{t}$ value indicates a higher norovirus load. $p$ values were calculated by the Kruskal-Wallis test, with Dunn's multiple comparison corrections. $C_{t}$, cycle threshold; NS, not significant; qRT-PCR, quantitative reverse transcription PCR. 
To validate the robustness of $\mathrm{C}_{\mathrm{t}}$ values, we randomly selected 80 samples (16 samples/season) according to quality control sampling scheme ANSI/ASQ Standard Z1.4 (https://asq.org) for repeat qRT-PCR measurement and inhibition study. We found a strong association between initial and repeat measurements (Spearman $r=0.82$; $\mathrm{p}<0.0001$ ) (Appendix Figure 3). Most samples gave an ideal $\mathrm{C}_{\mathrm{t}}$ difference of $\approx 1$ between undiluted and 2-fold diluted templates (median $\mathrm{C}_{\mathrm{t}}$ difference [IQR] 1.0 [0.9-1.1]), indicating minimal to mild inhibition (Appendix Figure 4). To exclude the possibility of genotype-specific quantification artifacts, we inspected the amplification efficiency of the assay by testing on 5-fold serial dilution of 3 strains for each virus genotype. The qRT-PCR efficiency in GII.Pe-GII.4 (100.8 \pm 5.3\%) and GII.P17-GII.17 (99.1 $\pm 2.6 \%)$ was equivalent to that of GII.P16-GII.2 $(95.0 \pm$ $4.3 \%)(\mathrm{p}=0.296$ by 1 -way ANOVA). We randomly selected 13 of 72 samples with low viral load $\left(C_{t}>25.0\right)$ for primers/probe sequence mismatch analysis; mismatch was noted in only 1 case, indicating that $\geq 96 \%$ samples with low viral load were free of primers/probe mismatch.

\section{Conclusions}

We found that GII.P16-GII.2 shed in higher amounts than pandemic GII.Pe-GII.4 in different age groups. This new strain, which is as replication competent as pandemic GII.Pe-GII.4, may cause severe gastroenteritis and lead to poor clinical outcomes (13). Our findings imply that the absence of prior exposure to this newly emerged strain may result in the delayed immune response and viral clearance in most populations. Immune naivety may be attributed to equally high viral loads of GII.P16-GII.2 and GII.Pe-GII.4 in children (1), providing a virologic explanation for the recent upsurge in the number of outbreaks caused by GII. P16-GII.2 in nursery schools, kindergartens, and elementary schools in Japan in the winter of 2016-17 (14). That report found a higher reproductive number of GII.P16-GII.2 compared with the previous 4 seasons, during which other norovirus genotypes, such as GII.Pe-GII.4, predominated, a result consistent with our findings of prominent viral load of GII.P16-GII.2 in children.

Our findings agree with a previous phylogenetic analysis, which showed that the capsid of GII.P16-GII.2 was closely related to earlier GII.2 strains, when it was speculated that its recent emergence may be attributable to high replication efficiency (6). Furthermore, recombinants carrying GII.P16, including GII.P16-GII.4 and GII.P16-GII.2, have caused $>60 \%$ of norovirus outbreaks in 2016 and 2017 in the United States (CaliciNet, https://www.cdc. gov/norovirus/reporting/calicinet/data.html). We propose that this time, rather than acquiring a new capsid variant, a new polymerase variant GII.P16 may be affecting norovirus epidemiology worldwide. This emerging and actively recombining norovirus polymerase genotype GII.P16 is highly transmissible, with pandemic risk. The mechanism behind replication difference among norovirus genotypes needs to be further studied by a virus cultivation system such as human intestinal enteroids (15).

Our study has limitations. First, we did not evaluate the viral load of GII.P16-GII.4 because this recombinant was sporadically $(n=21 ; 1.7 \%)$ observed. Second, we did not perform multivariate analysis to control for other confounding factors such as time from symptom onset to sample collection (viral load decreases over time) because of incomplete information, which may have introduced bias.

In summary, our results show that the emerging recombinant norovirus GII.P16-GII.2 is as replication competent as pandemic genotypes, which explains its high transmissibility and widespread circulation. Norovirus GII.P16-GII.2 has pandemic potential.

\section{Acknowledgments}

We thank Jenny Chan for providing proofreading and editorial assistance.

This study was supported in part by a grant from the commissioned Health and Medical Research Fund of Food and Health Bureau of the HKSAR Government (CU-15-C2, to M.C.-W.C.).

\section{About the Author}

Dr. Cheung is a postdoctoral fellow in the Department of Microbiology of the Chinese University of Hong Kong. Her research interests are norovirus epidemiology and transmission, and the use of advanced technologies such as enteroid model to study norovirus pathogenesis.

\section{References}

1. Bányai K, Estes MK, Martella V, Parashar UD. Viral gastroenteritis. Lancet. 2018;392:175-86. http://dx.doi.org/ 10.1016/S0140-6736(18)31128-0

2. Kroneman A, Vega E, Vennema H, Vinjé J, White PA, Hansman G, et al. Proposal for a unified norovirus nomenclature and genotyping. Arch Virol. 2013;158:2059-68. http://dx.doi.org/ 10.1007/s00705-013-1708-5

3. Cannon JL, Barclay L, Collins NR, Wikswo ME, Castro CJ, Magaña LC, et al. Genetic and epidemiologic trends of norovirus outbreaks in the United States from 2013 to 2016 demonstrated emergence of novel GII.4 recombinant viruses. J Clin Microbiol. 2017;55:2208-21. http://dx.doi.org/10.1128/JCM.00455-17

4. Ao Y, Wang J, Ling H, He Y, Dong X, Wang X, et al. Norovirus GII.P16/GII.2-associated gastroenteritis, China, 2016. Emerg Infect Dis. 2017;23:1172-5. http://dx.doi.org/10.3201/eid2307.170034

5. Bidalot M, Théry L, Kaplon J, De Rougemont A, Ambert-Balay K. Emergence of new recombinant noroviruses GII.p16-GII.4 and GII.p16-GII.2, France, winter 2016 to 2017. Euro Surveill. 2017;22:30508. http://dx.doi.org/10.2807/1560-7917. ES.2017.22.15.30508

6. Tohma K, Lepore CJ, Ford-Siltz LA, Parra GI. Phylogenetic analyses suggest that factors other than the capsid protein play a 
role in the epidemic potential of GII.2 norovirus. mSphere. 2017; 2:e00187-17. http://dx.doi.org/10.1128/mSphereDirect.00187-17

7. van Beek J, de Graaf M, Al-Hello H, Allen DJ, Ambert-Balay K, Botteldoorn N, et al.; NoroNet. Molecular surveillance of norovirus, 2005-16: an epidemiological analysis of data collected from the NoroNet network. Lancet Infect Dis. 2018;18:545-53. http://dx.doi.org/10.1016/S1473-3099(18)30059-8

8. Wang C, Ao Y, Yu J, Xie X, Deng H, Jin M, et al. Complete genome sequence of a novel recombinant GII.P16-GII.1 norovirus associated with a gastroenteritis outbreak in Shandong Province, China, in 2017. Genome Announc. 2018;6:e1483-17. http://dx.doi.org/10.1128/genomeA.01483-17

9. Chan MC, Leung TF, Chung TW, Kwok AK, Nelson EA, Lee N, et al. Virus genotype distribution and virus burden in children and adults hospitalized for norovirus gastroenteritis, 2012-2014, Hong Kong. Sci Rep. 2015;5:11507. http://dx.doi.org/10.1038/srep11507

10. Kwok K, Niendorf S, Lee N, Hung TN, Chan LY, Jacobsen S, et al. Increased detection of emergent recombinant norovirus GII. P16-GII.2 strains in young adults, Hong Kong, China, 2016-2017. Emerg Infect Dis. 2017;23:1852-5. http://dx.doi.org/10.3201/ eid2311.170561

11. Kageyama T, Kojima S, Shinohara M, Uchida K, Fukushi S, Hoshino FB, et al. Broadly reactive and highly sensitive assay for Norwalk-like viruses based on real-time quantitative reverse
transcription-PCR. J Clin Microbiol. 2003;41:1548-57. http://dx.doi.org/10.1128/JCM.41.4.1548-1557.2003

12. Shioda K, Barclay L, Becker-Dreps S, Bucardo-Rivera F, Cooper PJ, Payne DC, et al. Can use of viral load improve norovirus clinical diagnosis and disease attribution? Open Forum Infect Dis. 2017;4:ofx131. http://dx.doi.org/10.1093/ofid/ofx131

13. Desai R, Hembree CD, Handel A, Matthews JE, Dickey BW, McDonald S, et al. Severe outcomes are associated with genogroup 2 genotype 4 norovirus outbreaks: a systematic literature review. Clin Infect Dis. 2012;55:189-93. http://dx.doi.org/10.1093/cid/ $\operatorname{cis} 372$

14. Sakon N, Komano J, Tessmer HL, Omori R. High transmissibility of norovirus among infants and school children during the 2016/17 season in Osaka, Japan. Euro Surveill. 2018;23:18-00029. http://dx.doi.org/10.2807/1560-7917.ES.2018.23.6.18-00029

15. Ettayebi K, Crawford SE, Murakami K, Broughman JR, Karandikar U, Tenge VR, et al. Replication of human noroviruses in stem cell-derived human enteroids. Science. 2016;353:1387-93. http://dx.doi.org/10.1126/science.aaf5211

Address for correspondence: Martin Chi-Wai Chan, 1/F, Department of Microbiology, Lui Che Woo Clinical Sciences Building, Prince of Wales Hospital, Shatin, Hong Kong, China; email: martin.chan@cuhk.edu.hk

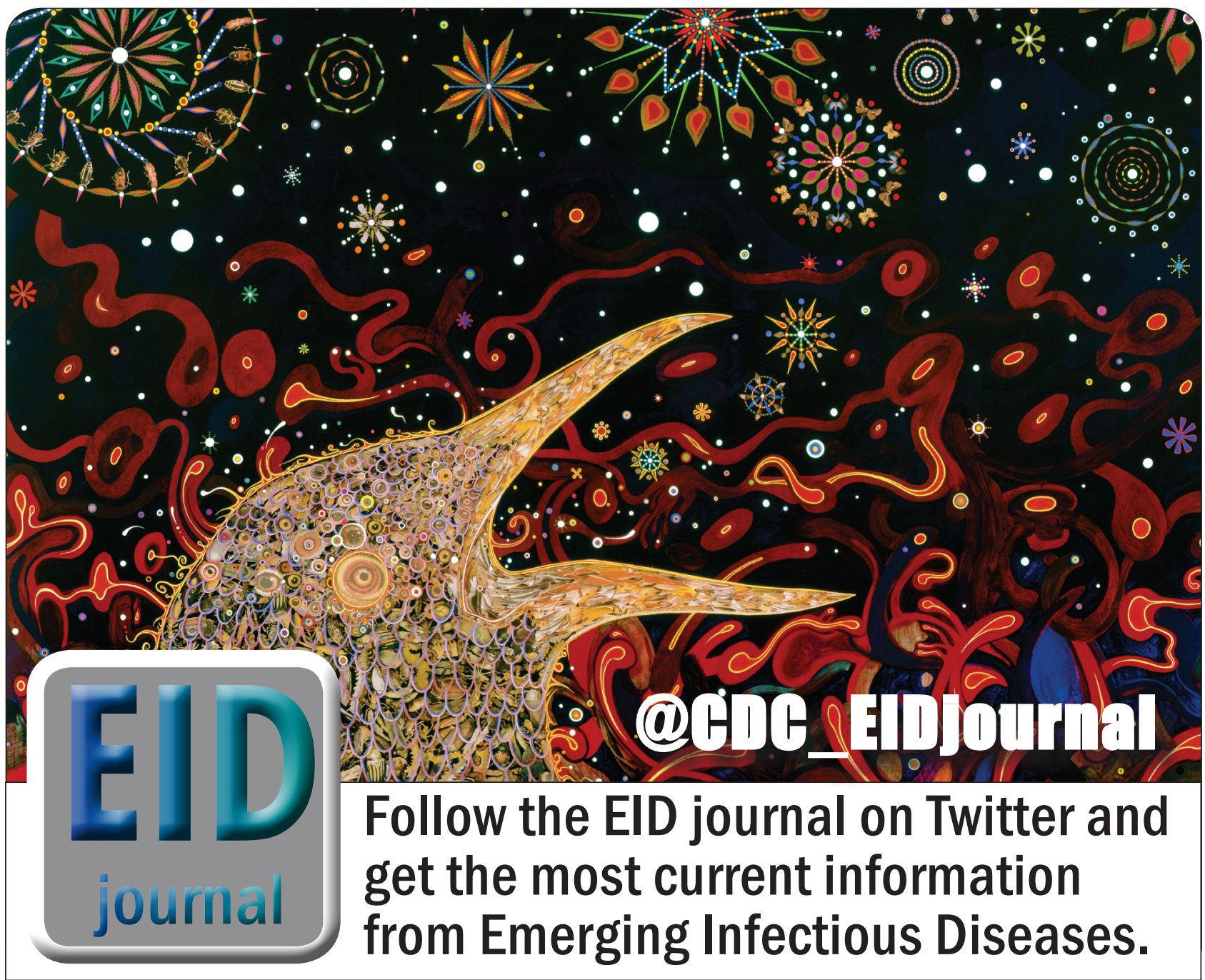

